\title{
1966: LES PROBLEMES DE LINGUISTIQUE GENERALE D'ÉMILE BENVENISTE
}

\section{6: PROBLEMS IN GENERAL LINGUISTICS BY ÉMILE BENVENISTE}

\author{
Irène Fenoglio \\ École Normale Supérieure, Institut des Textes et Manuscrits Modernes \\ (ITEM, UMR 8132 CNRS/ENS), Paris, França
}

\begin{abstract}
Résumé: Le recueil Problèmes de linguistique générale de 1966, en quantité d'articles, représente peu par rapport à l'ensemble de la production scientifique d'Emile Benveniste qui est immense, même arrêtée à 1965 mais il est extrêmement important par sa visée et par sa portée. L'érudition savante, l'apparent éclatement du savoir entre la connaissance approfondie de divers groupes de langue et mêmes de diverses langues est prise en charge dans une perspective linguistique de grande envergure et d'explicitation générale ; elle est aussi engagée dans une réflexion d'ordre épistémologique et anthropologique.
\end{abstract}

Mots clés: erudition; épistémologie; anthropologie; connaissance des langues.

Abstract: The compilation of Problems in General Linguistics of 1996 in several articles is small in relation to the whole scientific production of Émile Benveniste, which is huge, even with his arrest in 1965, but it is extremely important for its focus and scope. The erudition, the apparent emergence of knowledge between the deep knowledge of various language groups and even different languages is taken into account in a linguistic perspective of major and general relevance. It is also engaged in an epistemological and anthropological reflection.

Keyword: erudition; epistemology; anthropology; languages knowledge.

Jean-Claude Milner remarque que « la biographie d'Émile Benveniste (1902-1976) traverse plusieurs couches de l'histoire de la France du XXème siècle : les communautés juives d'Europe ; l'École linguistique de Paris ; le structuralisme ; le déclin des institutions intellectuelles de langue française " (MILNER, 2002, p. 87). La vie de Benveniste passe aussi par une fin dont le moins que l'on puisse dire est qu'elle fut difficile; de fait, elle fut tragique : sept longues années de paralysie et d'aphasie.

Trois ans avant de subir l'attaque cérébrale qui l'immobilisera définitivement, dix ans avant sa mort, Benveniste publie Problèmes de linguistique générale. Unique volume sous ce nom jusqu’à ce qu'en 1974 soit 
publié un second volume à partir duquel il sera nécessaire de préciser $P L G$ 1 (pour le volume de 1966) ou PLG 2 (pour celui de 1974). Benveniste est alors directeur d'études à l'Ecole Pratique des Hautes Études - il l'est depuis 1927 (avec interruption de 1940 à 1945 : obligation lui est faite de quitter son poste " pour appartenance à la race juive ", selon les termes utilisés par le gouvernement de Vichy, dirigé par P. Pétain) - et professeur au Collège de France depuis 1937 (avec la même interruption) où il occupe la chaire de " Grammaire comparée ». Dans les deux cas, il succède à Antoine Meillet.

\section{Publication des Problèmes de linguistique générale}

Jean-Claude Coquet ${ }^{1}$ se souvient des années 1960, précédant la sortie du volume :

Un point d'histoire. Quand j'ai suivi les cours à la Sorbonne pour le certificat de "Grammaire et philologie» et obtenu l'agrégation de grammaire, les noms de Benveniste et de Saussure étaient connus des étudiants, mais ce n'étaient que des noms. C'est R.L. Wagner qui m’a incité à lire Saussure... en 1960. Mais le «Meillet et Vendryes» (Traité de grammaire comparée des langues classiques) m'avait séduit au point que j'avais inscrit un sujet de thèse avec mon excellent professeur de latin, J. Perret, sur l'histoire de l'accusatif. Quant à Benveniste, il restait dans les marges, comme Saussure. J'ai attendu 1965, j'étais alors assistant à la Faculté de Poitiers, pour suivre ses cours au Collège de France. Sans interruption, et avec passion, de 1965 à 1969. En 1965-1966, si mes souvenirs sont exacts, dans une petite salle rectangulaire (une douzaine de personnes), puis, dans un grand amphithéâtre, dès 19661967. L'épopée structuraliste était lancée.

Pierre Nora confirme cette dernière remarque en évoquant la façon dont s'est faite la publication des Problèmes de linguistique générale et montre combien ce livre, et spécifiquement ce premier volume, demeure quasiment un best seller linguistique A déplacer :

En vérité, j’avais trouvé cet ouvrage engagé chez Gallimard à la rentrée 1965 pour la «Bibliothèque de philosophie» que depuis la mort de Merleau-Ponty dirigeait Pierre Verstraeten. Il me l'a cédé sans difficulté aucune pour que l'ouvrage rejoigne la première moisson de la «Bibliothèque des sciences humaines». Comme je comptais publier en même temps Les mots et les choses

\footnotetext{
${ }^{1}$ Je remercie chaleureusement Jean-Claude Coquet, Pierre Nora et Julia Kristeva (ordre d'arrivée dans le texte) qui ont répondu très positivement, comme on pourra en juger, à ma demande d'apporter un témoignage sur ce que la publication du premier volume des Problèmes de linguistique générale avait représenté pour eux.
} 
de Michel Foucault et Ethnologie et langage de Geneviève Calame-Griaule, l'ensemble faisait un tir groupé autour des Problèmes du langage. Il était sûr que la publication de Benveniste dans ce cadre prendrait davantage de relief et servirait aussi le lancement de la collection. C'est ce qui s'est passé. L'ouvrage a eu un succès immédiat. Je me souviens de la stupeur de Benveniste quand je lui ai annoncé la réédition quelques mois plus tard et sa surprise de voir son cours et son séminaire du Collège de France connaître une affluence inattendue.

Cet événement que constitue la publication des $P L G 1$ est attesté aussi par Mohamed Djafar Moïnfar qui, dans l'avant-propos du second volume - qu'il a édité avec l'accord et la relecture d'Emile Benveniste, alors alité, paralysé et aphasique depuis cinq ans - s'exprime ainsi : "L'immense intérêt porté aux Problèmes de linguistique générale, traduits assez tôt en anglais, en italien et en espagnol, a suscité de la part de bon nombre d'amis et élèves le souhait que cette entreprise ait une suite et que paraisse un nouveau volume».

Il faut relire les propos enthousiastes de Roland Barthes, à la sortie des PLG ; il signe trois pages dithyrambiques, le 15 mai 1966, dans $L a$ quinzaine littéraire. Après avoir évoqué l'ensemble du recueil, Roland Barthes s'exclame :

Tout cela forme le bilan d'un savoir impeccable, répond avec clarté et force aux questions de fait que tous ceux qui ont quelque intérêt pour le langage peuvent se poser. Mais ce n'est pas tout. Ce livre ne satisfait pas seulement une demande actuelle de la culture : il la devance, il la forme, la dirige. Bref, ce n'est pas seulement un livre indispensable ; c'est aussi un livre important, inespéré : c'est un très beau livre (BARTHES, 1966, p. 815).

Il ajoute quelques lignes plus loin :

[...] il est capital pour tout un ensembles de recherches et de révolutions qu'un linguiste aussi rigoureux que Benveniste soit lui-même conscient des pouvoirs de sa discipline, et que, refusant de s'en constituer le propriétaire, il reconnaissait en elle le germe d'une nouvelle configuration des sciences humaines (BARTHES, 1966, p. 815).

Le recueil Problèmes de linguistique générale de 1966, en quantité d'articles, représente peu par rapport à l'ensemble de la production scientifique d'Emile Benveniste qui est immense, même arrêtée à $1965^{2}$

\footnotetext{
${ }^{2}$ Voir Djafar Moïnfar, Bibliographie d'Émile Benveniste, in Mélanges linguistiques offerts à Émile Benveniste, Paris, Louvain, Société linguistique de Paris-Peeters, 1975, p. IX-LIII.
} 
mais il est extrêmement important par sa visée et par sa portée. L'érudition savante, l'apparent éclatement du savoir entre la connaissance approfondie de divers groupes de langue et mêmes de diverses langues est prise en charge dans une perspective linguistique de grande envergure et d'explicitation générale ; elle est aussi engagée dans une réflexion d’ordre épistémologique et anthropologique.

\section{Linguistique générale}

La linguistique générale a toujours été la visée stable de Benveniste. Véritable enjeu théorique et plus précisément épistémologique, elle est demeurée la préoccupation sous-jacente à toutes ses descriptions de langues diverses et variées et à toutes les analyses de détail du fonctionnement des faits langagiers. Si l'on parcourt les résumés de ses cours au Collège de France, on s'aperçoit que sous la dénomination inchangée de la chaire qu'il occupait - chaire de " grammaire comparée " - il faisait deux séries de cours. Pour le "cours du lundi " il qualifiait toujours l'objet d'étude choisi (par exemple, le fonctionnement de la syntaxe) de " général ». Une étape de plus est franchie à partir de l'année 1963-1964 : sous un titre de chaire toujours inchangé, " grammaire comparée ", il précise comme intitulé général de ses cours du lundi «Problèmes de linguistique générale » : 


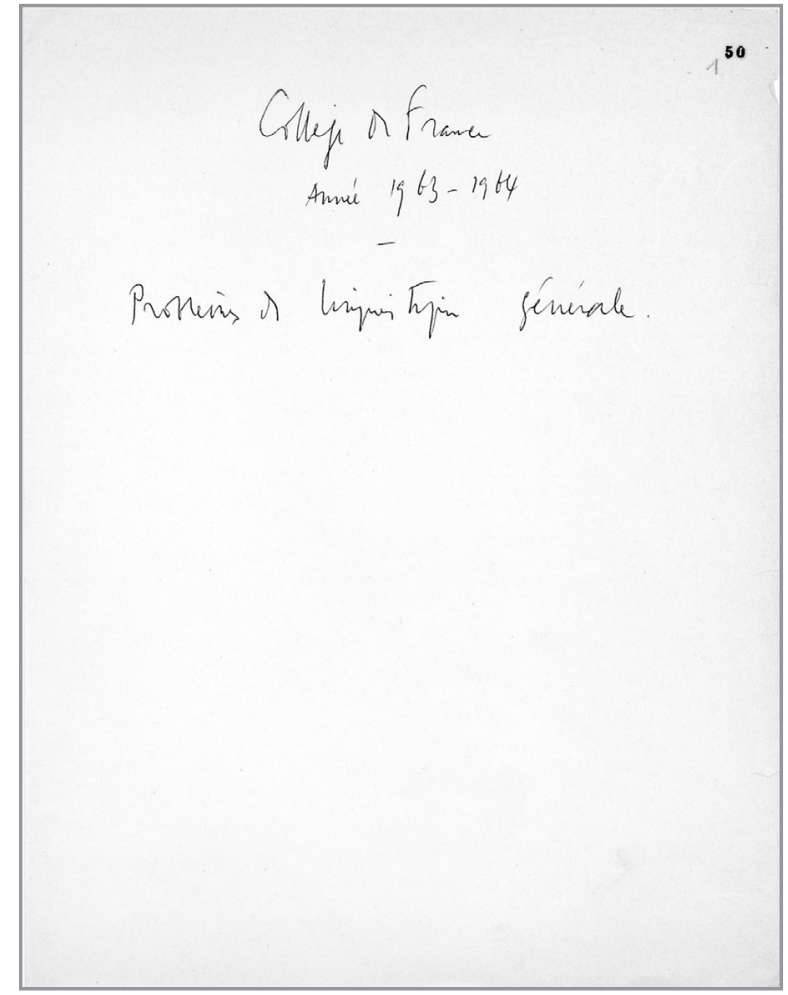

Fonds Benveniste de la BnF, Pap Or, boite 43, env. 105, f 50.

Voici comment il débute le résumé des cours de cette année là:

Les problèmes de linguistique générale, auxquels nous avons consacré les leçons du lundi, sont ceux que Saussure a le premier proposés à la réflexion. Nous avons considéré cet objet singulier qu'est devenu la langue depuis que Saussure, en une prise de conscience historique, l'a restituée dans sa nature propre, et nous avons essayé de discerner ce qui en fait quelque chose d'irréductible à tout ordre de phénomènes (Annuaire du Collège de France, Année 1964, p. 289).

Cette visée de linguistique générale ne cessera plus d'être mentionnée pour ses cours "du lundi " au Collège de France, les "leçons du mardi " restant consacrées au domaine indo-européen, indo-iranien ou autre langue ou ensemble de langues : sémitiques, caucasiennes, amérindiennes...etc., pour lesquelles il n’a jamais hésité à aller faire des enquêtes de terrain et 
parfois dans des conditions difficiles.

"Problèmes de linguistique générale " : cette expression n'est donc pas née pour la publication de 1966, elle est l'expression d'une profonde et longue réflexion, mûrie durant la préparation de ses cours, devant l'héritage et de Saussure et de Meillet. "Linguistique générale " renvoie à l'émergence d'un champ qui, peu à peu, deviendra une discipline. L'expression est sinon apparue du moins circule avec le Cours de linguistique générale de Ferdinand de Saussure (publié en 1916), elle s'affirme avec la Linguistique historique et linguistique générale d'Antoine Meillet (publié en 1921). La linguistique générale offre une nouvelle orientation aux recherches de grammaire comparée en ouvrant un espace à la théorie générale sur la langue, en développant et amplifiant les travaux de grammaire historique et comparée des langues indo-européennes ainsi que d'autres types de langues. Il s'agit d'une linguistique théorique qui s'interroge sur la constitution et l'organisation même $d u$ linguistique et sur les méthodes d'analyses permettant de le faire apparaître.

Ainsi, par exemple, en 1954 sort un article "Forme et sens de mnaomai $i^{3}$ ", article spécifique, étude très particulière, mais le cadre méthodologique "forme et sens" sera repris pour un article de linguistique générale "Forme et sens dans le langage " (PLG 2) qui s'interroge sur la façon dont ce rapport peut être pensé et se propose de le structurer de façon à ce qu'il puisse comprendre les faits de langue en général.

La linguistique générale rend compte, pour Benveniste de la façon dont on peut comprendre le fonctionnement de la langue, saussurienne, à partir des processus d'actualisation propres à chaque langue particulière. "Linguistique générale » désigne alors, en quelque sorte, le rapport entre les langues et la langue, ou, dit autrement, entre les langues et le linguistique; elle constitue, selon lui, en juste héritier de Saussure, l'essence même de l'activité linguistique.

Voici comment il présente la question dans son cours de 1963-1964:

${ }_{3}$ " Formes et sens de $\mu v \alpha o \mu \alpha \mathrm{l}$ ", Festschrift Albert Debrunner, 1954, p. 13-18. 


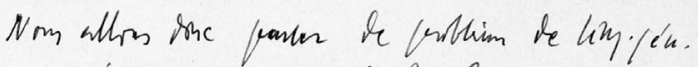

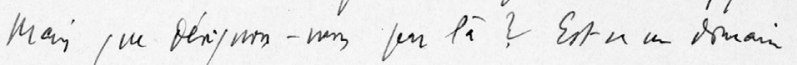

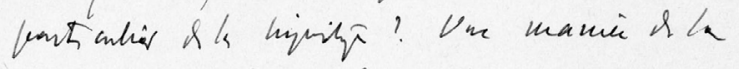
venive? An pari?

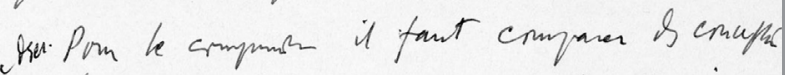

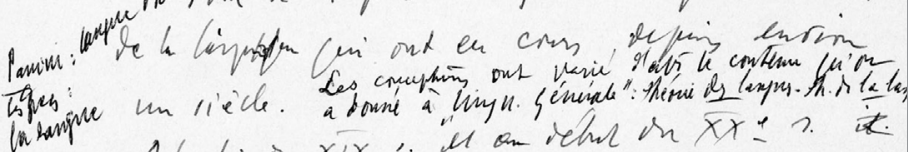
Ah fir on $x / x$. "N an ichol on $x x \geq 1$.

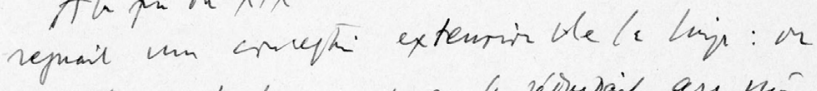

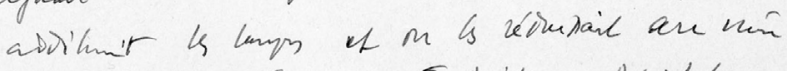
Demomi. he thaserie F.Mulls, Mitteli ets. mesmaient lint a la minu acrue le ferre,

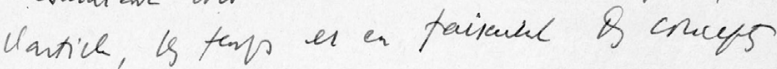

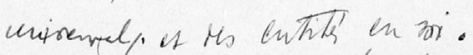

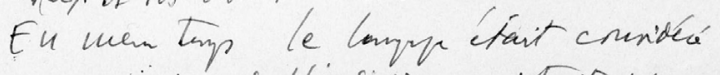

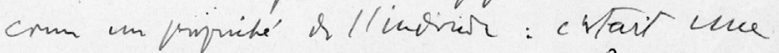
certain ta cuclté boychine. H. Paul frockmail que ln lampue n'oxistait pes $h_{n}$ os

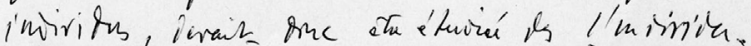

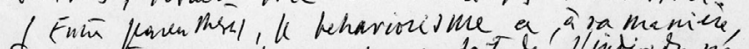

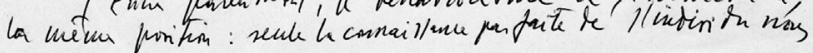

Fonds Benveniste de la BnF, Pap or, 43, env. 105, $f^{\circ} 70$.

Mais dans "Problèmes de linguistique générale » il y a deux items : celui de "linguistique générale » et celui de "problèmes ».

"Problèmes " - renvoie à l'idiosyncrasie réflexive et méthodologique de Benveniste qui consiste toujours à aborder un fait de langage sous forme de questions, le terme "problème " ajoutant à celui de " question " la dimension d'une complexité non encore résolue. Cela est visible dans ses publications où le mot " problème " est très présent, mais cela l'est 
encore plus dans ses manuscrits, notes et brouillons ${ }^{4}$, où nous voyons Emile Benveniste aux prises avec le désarroi du chercheur, ne sachant par où commencer sa réflexion :

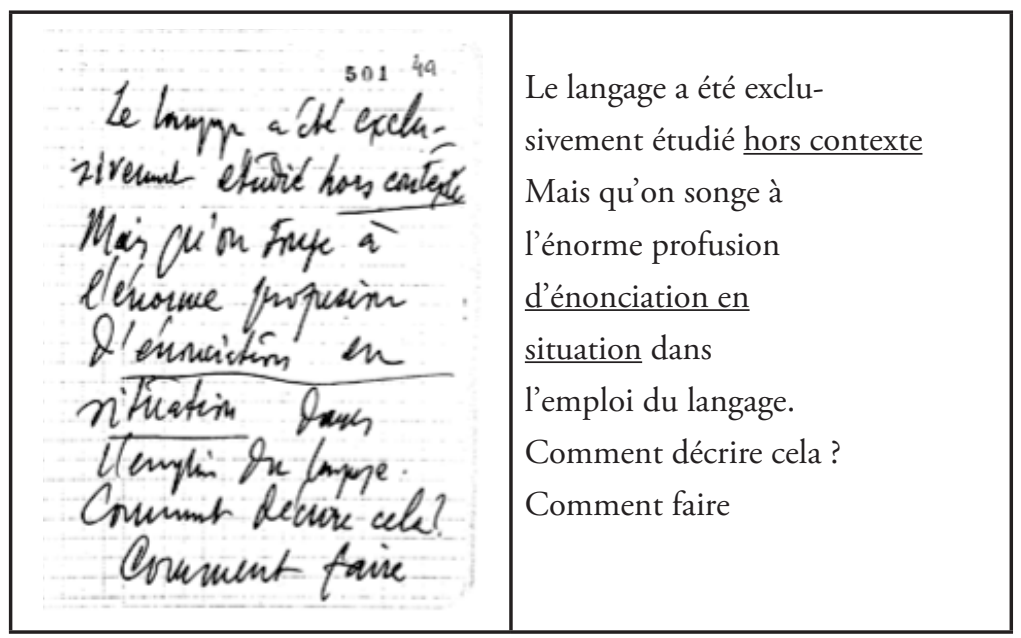

avec les tentatives ( essayer») pour poser sa pensée ${ }^{5}$

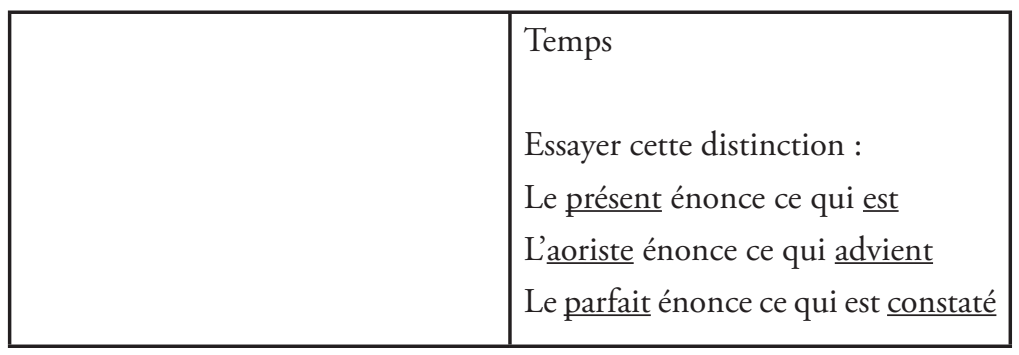

avec l'émotion devant la découverte dont la frontière est toujours si ténue entre l'évidence et l'étonnement

\footnotetext{
${ }^{4}$ Nous rappelons que c’est Benveniste, lui-même, qui, de son vivant, à légué, par testament, l'ensemble de ses papiers de travail à la Bibliothèque nationale (voir "Les papiers d'Emile Benveniste " par Émilie Brunet, in Émile Benveniste, Dernières leçons. Collège de France 1968 et 1969, éd. EHESS, Gallimard, Seuil, 2012, p. 175-180).

${ }^{5}$ Note préparatoire au cours aux Collège de France 1963-1964, intitulés « cours de linguistique générale », Fonds Benveniste BnF, Pap Or, boite 43, env. 104, f²6.
} 


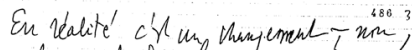

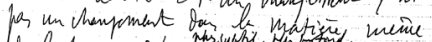

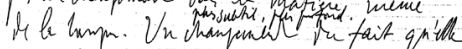
est mise in mowe went, cre juitgn'm r'en sn empari et qu'ta ment, he mech in action, que at appacil qui. gidait, potentid, mai inute, conentant

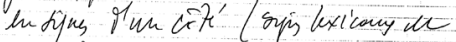
auty) in modets texiounch at som

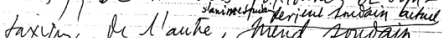

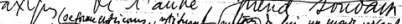
existence, Sictipe chre nait an monde alto. Vh houme

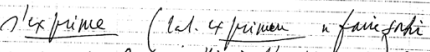
in premant, fain joillis: I'extérieur T), il fait juillir bu langue daus l'exonciason

Fonds Benveniste de la BnF, Pap. Or. 51, env. $198, f^{\circ} 486$.
En réalité c'est un changement, non, pas un changement dans la matière même de la langue. Un changement <plus subtil, plus profond> du fait qu'elle est mise en mouvement, que quelqu'un s'en est emparé et qu'elle la meut, la met en action, que cet appareil qui gisait, potentiel, mais inerte, consistant en signes d'un côté (signes lexicaux et autres), en modèles flexionnels et syntaxiques de l'autre <'anime soudain devient soudain actuel> prend soudain existence $<$ se forme en discours restituant autour de lui un mouvement vivant> ; te langure. Quelque chose naît au monde alors. Un homme s'exprime (lat. exprimere "faire sortir en pressant, faire jaillir à l'extérieur"), il fait jaillir la langue dans l'énonciation

Nous pourrions continuer les exemples, ils ne manquent pas ; Benveniste, traduit et maîtrise, par l'emploi de ce terme " problème ", la recherche insatiable à laquelle il est en proie devant les faits de langage. "Problèmes ", donc ; tout est "problème " lorsqu'on s'interroge sur le langage ${ }^{6}$. Du reste, il insiste lui-même dans l'Avant-propos des $P L G:$ :Si on les [les études réunies dans cet ouvrage] a présentées sous la dénomination "problèmes ", c'est qu'elles apportent dans leur ensemble et chacune pour soi une contribution à la grande problématique du langage, qui s'énonce dans les principaux thèmes traités : on y envisage les relations entre le biologique et le culturel, entre la subjectivité et la sociabilité, entre le signe

\footnotetext{
${ }^{6}$ C. Puech remarque : "Problèmes", "problématique" : s'il y a des styles de pensée, et si il y a un style de pensée proprement benvenistien, c'est bien celui de la "problématisation ". Il s'agit avant tout d'ouvrir des perspectives, de mettre en relation, de définir des points de vue et pour cela de ne pas se satisfaire des découpages disciplinaires certes éprouvés, mais aussi institués. De ce point de vue, les PLG ne manquent jamais une occasion de remettre en cause l'autosuffisance des savoirs linguistiques qui s'accordent d'emblée la consistance disciplinaire dont ils devraient au contraire apporter la preuve. " ("Benveniste et la représentation de la discipline linguistique ", Linx 9 : Émile Benveniste, vingt ans après, 1997.)
} 
et l'objet, entre le symbole et la pensée, et aussi les problèmes de l'analyse intralinguistique »

En forme d'avertissement, Benveniste précise : " [À] ceux qui découvrent dans d'autres domaines l'importance du langage $[. .$.$] certaines$ pages pourront sembler difficiles. Qu'ils se convainquent que le langage est bien un objet difficile et que l'analyse du donné linguistique se fait par des voies ardues"

Autrement dit, Benveniste oppose une exigence de complexification, une vigilance de non édulcoration de la difficulté, à une linguistique de modélisation a priori dont on pourrait penser que la simplification qu'elle promeut, elle aussi a priori, nous permettrait de comprendre les faits de langage.

Dans ce premier volume de linguistique générale, Benveniste expose l'ampleur de son champ. Il l'organise en six chapitres: I. Transformations de la linguistique, II. La communication, III. Structures et analyses, IV. Fonctions syntaxiques, V. L'homme dans la langue, VI. Lexique et culture. On peut y lire une organisation pensée, une composition réfléchissant le mouvement théorique dans lequel s'engage Benveniste. Le premier article est un état des lieux : "Tendances récentes en linguistique générale ", le dernier est un rappel de la contribution linguistique à la compréhension de la socialité humaine : "Civilisation : contribution à l'histoire du mot ». Entre ce tenant et cet aboutissant, quatre chapitres s'attachent à "structurer " et méthodologiser le linguistique.

Ce volume témoigne aussi de l'attention curieuse de Benveniste aux nouvelles perspectives ouvertes par les recherches psychanalytiques. Il faut citer "Remarques sur la fonction du langage et la découverte freudienne " qui s'y confronte directement, mais voir surtout comment cette « découverte freudienne " est prégnante dans la recherche rigoureuse que Benveniste entreprend pour repérer et caractériser linguistiquement les marques de la subjectivité dans l'usage du langage ${ }^{7}$; plusieurs articles particuliers des $P L G 1$ peuvent être cités relevant directement de cette problématique : en particulier les articles 18 "Structures des relations de personnes dans le verbe ", 19 "Les relations de temps dans le verbe français ", 20 "La nature des pronoms ", 21 " De la subjectivité dans le langage ", tous faisant partie du chapitre V intitulé "L'homme dans la langue ». Il s'agit alors de " restituer dans le langage la non-homogénéité et la non-unicité réelle de la subjectivité parlante [...] justement ce que, par son expérience propre, la

${ }^{7}$ Ce que Lacan n'a pas voulu voir (ou dont il n'a rien dit) dans sa critique, un peu trop rapide de Benveniste ne mentionnant que l'article « Remarques... ». 
théorie du désir avait dû accomplir " ainsi que le constatent Judith et JeanClaude Milner ${ }^{8}$. Il s'agit de disjoindre l'individuel de la subjectivité et de « fonder le sujet et la subjectivité sur une base matérielle : le langage en tant qu'il se profere " (MILNER, 2002, p. 93). Du reste, Julia Kristeva, confirme cette lecture ci-après.

Le fonds Benveniste de la BnF possèdent plusieurs ensembles de Manuscrits correspondant à des articles des PLG $1^{9}$ mais, malheureusement ne s'y trouvent pas ceux correspondant à "De la subjectivité dans le langage " que nous aimerions tant consulter.

Ce plan des Problèmes de linguistique générale a été repris pour les PLG 2. Jean-Claude Coquet expose la façon, dont, à ses yeux, les deux volumes des $P L G$ sont indissociables.

Les cours que j'ai suivis (1965-1969) ont fourni la matière des PLG II. Le Lexique d'É. Benveniste, en deux fascicules, publié par l'Université d'Urbino en 1971 et 1972, a été constitué à partir des PLG I, que j'ai donc dépouillés avec soin dès 1967. J'ai été accompagné dans cette entreprise par un jeune Belge de talent, Marc Derycke. Pour la confection de ce lexique, Benveniste m'avait conseillé de prendre pour modèle les lexiques publiés par le Comité internationnal permanent des linguistes, tels le Dictionnaire de linguistique de l'École de Prague, le Lexique de la terminologie saussurienne ou le Glossaire de E P. Hamp. Il m’avait donné quatre tirés à part pour m'aider à mettre à jour le lexique. Trois d'entre eux ont trouvé leur place dans les PLG II ; le quatrième a été publié en 1965 par la revue d'anthropologie qu'il dirigeait avec C. Lévi-Strauss, L'Homme. Cette même revue m’avait demandé imprudemment de faire la recension du Vocabulaire des institutions indoeuropéennes publié par les Éditions de Minuit en 1969. Je ne l'ai pas faite, par manque de compétence à cette époque. Mais je me suis imprégné peu à peu du Vocabulaire et de cet autre magnifique petit livre (sa seconde thèse), Noms d'agent et noms d'action en indo-européen, qui me sont devenus aussi familiers que les deux tomes, à mes yeux, indissociables des PLG.

\section{Fortune et actualité des Problèmes de linguistique Générale}

Julia Kristeva témoigne, pour sa part, non seulement de son enthousiasme mais aussi de l'influence sur sa pensée de la lecture des PLG:

\footnotetext{
8 "Judith et Jean-Claude Milner, "Interrogations, reprises, dialogue " in Langues, discours, société. Pour Emile Benveniste, Seuil, 1975, p. 143.

${ }^{9}$ On peut se reporter au repérage des archives et brouillons d'Emile Benveniste correspondant aux articles des Problèmes de Linguistique Générale sur le site de l'équipe « Génétique et théories linguistiques de l'ITEM : http://www.item.ens.fr/index.php?id=200861
} 
Je ne connaissais pas l'œuvre de Benveniste avant mon arrivée en France. Mes études en Bulgarie comportaient un enseignement assez complet en "philologie " (du français et de l'anglais) basée essentiellement sur la phonétique, la lexicologie et la grammaire, et précédé par du grec et du latinauquel s'ajouta (au niveau du "doctorat ") un solide cursus philosophique (beaucoup de Hegel et un peu de Husserl) - mais très peu de linguistique générale (le Cours de Saussure essentiellement). C'est Roman Jacobson (il avait transité par l'université de Sofia dans sa jeunesse et, ami des futuristes puis d'Aragon, se montra très généreux envers l'étudiante bulgare "plongée " dans le langage des avant-gardes littéraires) qui m’a recommandé de lire les Problèmes de linguistique générale qui venaient de paraitre au début de 1966 (je suis arrivée à Paris à la veille de Noël 1965).

J'ai été séduite par quelques articles recueillis dans ce livre, avant de rencontrer Benveniste et d'approfondir ma connaissance de l'œuvre et de la personne, comme je l'évoque dans ma préface aux Dernières leçons ${ }^{10}$. Mais nous n'avons pas commenté son travail autrement que ce que j'évoque dans ma préface aux Dernières leçons.

- D'abord, "Nature du signe linguistique ". Saussure commençait à être lu, on discutait les Eléments de sémiologie (1964-65) de Barthes. Le texte de Benveniste a changé la donne : il montrait que ce n'est pas le "signe " qui est arbitraire, mais le rapport entre le référent d'une part et le signe (signifiant-signifié) de l'autre. Je ne pourrais ici développer les subtilités de ce débat. Je voudrais seulement insister sur son impact que je qualifierais d'épistémologique. Il révélait une manière de penser, au croisement de la linguistique et de la philosophie, qui ouvrait l'espace du signe et annonçait que celui-ci est susceptible d'interprétations et de modulations. Un autre type de linguiste était ici au travail, qui se faisait fort de saisir nos manières d'être au monde à travers les diverses logiques dont sont capables les signes linguistiques eux-mêmes. Plus tard, Benveniste devait introduire les notions de "sémiotique " et de "sémantique ". D’une autre façon, attentive à la théorie psychanalytique de l'inconscient et des pulsions, j'ai parlé de "sémiotique " et de " symbolique " (La Révolution du langage poétique, Seuil, 1974, p. 17-101). Mais l'impulsion venait de cette première audace : une véritable intrusion dans la citadelle saussurienne, et autrement que ne l'avait faite C. S. Pierce, car à partir de la chaîne signifiante des langues naturelles elles-mêmes. J'ouvre aujourd'hui le livre et je retrouve les pages désormais jaunissantes que j'avais soulignées au crayon : " Entre le signifiant et le signifié le lien n'est pas arbitraire ; au contraire, il est nécessaire..." "C'est en effet, transposé en terme linguistique, le problème métaphysique de l'accord entre l'esprit et le monde $[. .$.$] que le linguiste fera mieux pour$ l'instant de délaisser [...] le signe recouvre et commande la réalité ; mieux, il est cette réalité..."

- Plus encore, cette délimitation du champ de la linguistique, sous sa modestie apparente, révélait une exorbitante ambition : il s'agissait d'introduire dans la formalité de la chaine signifiante elle-même le sujet parlant! Et pas uniquement celui de Descartes qu'affectionnait Chomsky, mais une "subjectivité " d' "allocution " qui résonnait pour moi avec

${ }^{10}$ Émile Benveniste, Dernières leçons. Collège de France 1968 et 1969, éd. EHESS, Gallimard, Seuil, 2012, p. 13-40. 
certaines avancées phénoménologiques de Husserl voire du second Heidegger (celui du langage pris dans la proximité du dialogue). « La nature des pronoms " et " De la subjectivité dans le langage » ont été des révélations et, sans exagération, des phares pour ma réflexion en sémiologie du langage poétique, comme, je crois, pour nombre de chercheurs à cette époque et depuis... « Je » et " tu » se référant uniquement à la "réalité du discours »; " il " étant " membre non marqué de la corrélation de personne ", une " non-personne ", bien que dans les langues indo-européennes "il " semble membre d'un paradigme à trois termes... " Je rappelle ces éléments devenus aujourd'hui des truismes (du moins pour les linguistes) et qui m'avaient (nous avaient) révélé de manière indélébile combien « unique est la condition de l'homme dans le langage " (p. 260). Ils ont résonné pour moi avec ma relecture du "dialogisme " de Bakhtine que j'ai introduit en France puis aux USA, "Le mot, le dialogue et le roman ${ }^{11}$, où l'on trouve les notions "discours / histoire ", "sujet de l'énoncé/sujet de l'énonciation ", etc...

- Last but not least, cette "subjectivité " dans le langage n'ignorait pas "le langage dans la découverte freudienne ». Benveniste reste - à mon sens jusqu’à présent et malgré quelques essais de la part de "linguistes purs " (ce que je ne suis pas ) après lui - le seul linguiste qui a traité sérieusement de la place du langage dans la découverte freudienne. Différemment de Lacan (qui avait pourtant souhaité cette étude pour sa revue), Benveniste distingue le langage comme " une catégorisation, une création d'objets et de relations entre ces objets ", des logiques de l'inconscient. Car, tandis que "à la différence des signes linguistiques ", les "signifiants " dans le symbolisme des rêves sont liés par des rapports de "motivation ", de telle sorte que l'inconscient se révélerait davantage dans une "rhétorique " (que comme un "signifiant ") sous l'effet d'une "force anarchique que refrène ou sublime le langage normalisé » (p.78). Cette interprétation m’a beaucoup soutenue dans mon propre travail sur la "chora sémiotique " que j'envisage sous l'effet de la langue, mais articulée précisément par les pulsions inconscientes...

Des notions comme celle de "sujet de l'énonciation » ou la distinction " discours-histoire " ont été assimilées par d'autres chercheurs, sans que ces ouvertures - encore abyssales (côté psychanalyse, côté phénoménologie : tout reste à faire) - aient été prises en considération dans leur complexité. Ce travail me semble nécessaire, face aux diverses déconstructions de l'unité supposée des sujets parlants, telles qu'elles se présentent dans des situations de pathologies psycho-sexuelles ou encore sous l'assaut de l'image et de l'hyperconnexion.

- Je voudrais rappeler un dernier texte de ce recueil qui m'a beaucoup marqué et dont les conséquences ont été plus longues à s'imposer dans ma propre pensée : "La phrase nominale ", auquel j'ai très vite associé le livre de Benveniste Les infinitifs avestiques. La distinction nom/verbe : par delà la distinction objet/procès, le verbe est défini par Benveniste comme l'élément assertif et cohésif, tandis que la phrase nominale est assertive mais " hors de toute relation avec le locuteur ": elle pose l' " absolu " (non la situation) et le " discours" (pas la narration) : être VS existence ? Croire-foi-divin VS Savoir ? C'est seulement récemment, par mon écoute de certains "états limites » en psychanalyse, ou bien en interprétant le " discours mystique "

\footnotetext{
${ }^{11}$ Séméiotikè, Recherches pour une sémanalyse, p.171
} 
hors-de-soi, que ces textes de Benveniste me sont revenus, après m'avoir frappée à l'époque, mais sans suite, autre que la lecture de certains de ses travaux d'indo-européaniste...

Il me semble que les chercheurs attentifs à la psychanalyse lacanienne, et en discussion avec elle, comme Luce Irigaray, ont été les plus sensibles à ces dimensions du premier volume des Problèmes de linguistique générale. Je cite un de ses articles dans Cahiers pour l'analyse, et dans Séméiotikè, Recherches pour une sémanalyse ${ }^{12}$.

\section{Jean-Claude Coquet précise l'impact de la publication des PLG et précise diverses réactions :}

Que dire maintenant de l'impact scientifique des seuls $P L G$ I ? Je laisse donc de côté le succès éditorial considérable des PLG I dès sa sortie (plusieurs dizaines de milliers d'exemplaires vendus) ; il est comparable à celui des Mots et des choses. Je ne retiendrai ici que quelques éléments touchant à la linguistique structurale ou générative de cette époque.

L'accueil des linguistes a été très contrasté selon leur école de référence. Pour les "fonctionnalistes" (autour de Martinet), Benveniste est "peu créateur" ; ses "quelques suggestions" sont "nourries d'une philosophie très introspective du langage" (G. Mounin, Lingua, 1967). À l'inverse, pour les "générativistes" (autour de Chomsky), Benveniste (je suis N. Ruwet), est "de tous les linguistes structuralistes européens, celui qui, dans ses recherches concrètes, s'est montré le plus proche des conceptions de Chomsky". Pour preuves, par exemple, le traitement du génitif latin ou l'étude sur la phrase relative. J'ajoute que N. Ruwet cite les Cours au Collège de France 19631964 et 1965-1966 (Intoduction à la grammaire générative, Plon, 1967).

Pour autant, un enseignement tiré des PLG n'est pas entré facilement dans les Universités. C. Normand a noté que son département de linguistique de Nanterre ignorait Benveniste en 1966 et qu'il lui a fallu passer par le département de philosophie (P. Ricoeur) pour le connaître. Ricoeur s'appuie en particulier sur la "Structure des relations de personne dans le verbe" (1946), sur "La nature des pronoms" (1956) et sur "De la subjectivité dans le langage" (1958). Non sans réserves, car il voit (et il voit juste) dans l'entreprise de Benveniste un risque d'effraction de son propre domaine : la philosophie, qui n'est pas, assure-t-il, celui du linguiste ${ }^{13}$. Cette réserve est déjà celle de Mounin et se mue en condamnation quelques années plus tard sous la plume d'A. Culioli commentant en 1984 la même citation que Ricoeur : "Le langage seul fonde en réalité, dans $s a$ réalité qui est celle de l'être, le concept d' 'ego' “ (PLG I, p. 259). "Le recours à la notion d' 'être' n'arrange pas le commentaire"14. Mais justement, là est l'avancée. "Une certaine linguistique qui se prétend moderne ", comme le dira Benveniste

\footnotetext{
${ }^{12}$ Seuil, 1969, p. 157

${ }^{13}$ Esprit, mai 1967 , p. 812

${ }^{14}$ É. Benveniste aujourd'hui, tome 1, Bibliothèque de l'Information grammaticale, 1984, p. 83
} 
un peu plus tard, en $1968^{15}$, n'a d'autre objet que la linguistique formelle (le sémiotique). Or il ne faut pas occulter le prédicat de réalité. Benveniste s'est refusé à employer le terme honni de "substance ". Et pourtant, c'est bien de cela qu'il s'agit. Si l'on retient comme exemple (ce que fait Ricoeur) le traitement du pronom personnel "je", Benveniste pose qu'il y a deux "je", " deux instances conjuguées ${ }^{16}$ : l'une est une instance formelle, "linguistique", l'autre est une instance "personnelle", une présence. Ce dernier "je” relève d'un prédicat de réalité. Il "signifie la personne qui énonce la présente instance de discours contenant je ".

Il est vrai que ce qui paraît être un recours à l'ontologie n'est pas acceptable par les structuralistes. Notons au passage que si Benveniste pratique en scientifique l'analyse structurale, il n'adhère pas à l'idéologie structuraliste.

Rendons encore hommage à Ricoeur. Même s'il s'est refusé à suivre Benveniste sur le terrain qu'il dénomme " extralinguistique " (" la présupposition extralinguistique du pronom personnel ", loc.cit.), il a souligné le fait (p. 810), que "la phénoménologie du sujet parlant » trouverait " un appui solide dans les recherches de linguistes comme Émile Benveniste”."

Un dernier témoignage sur la réception de Benveniste après PLG I. A.-J. Greimas, à l'encontre de Mounin, salue l'« apport novateur " de Benveniste, mais se reprend-il aussitôt en bon structuraliste, cet apport novateur «a pu donner lieu, il est vrai, à de nombreuses exégèses d'ordre métaphysique ou psychanalytique, exaltant toutes les réapparitions inespérées du sujet, et permettant de refouler la conception "anonyme" du langage considéré - et déconsidéré - comme un système de contraintes ${ }^{17}$. Aspect novateur, certes, mais qu'il fallait savoir corriger.

En résumé, accueil bien ambigu des PLG I .

Et pourtant, quelle excellente et formidable décision que cette publication dont Benveniste dit, dans son Avant-propos, que l'initiative en revient à MM. P. Verstraeten et Nicolas Ruwet (reprise, comme nous le savons, par Pierre Nora), car la linguistique générale n'est pas seulement un enjeu pour Benveniste, c'est un enjeu pour la linguistique. C'est ce qui fait qu'un linguiste n'est pas un simple descripteur de langue mais un penseur qui tente d'éclairer de son point de vue complexe, très complexe, et depuis son savoir forcément érudit et cumulatif, ce qui se passe du fait que l'homme parle, du fait que l'animal homme a cette compétence étrange, extraordinaire et péremptoire qu'est l'exercice du langage ; et du fait que cette compétence l'engage dans des relations subtiles avec ses congénères et potentiellement d'une richesse incommensurable mais non nécessairement clarifiées.

Benveniste précise dans son Avant-propos des PLG 1 :

\footnotetext{
${ }^{15}$ manuscrit de l'article « La blasphémie et l'euphémie "

${ }^{16}$ PLG I, p. 252

${ }^{17}$ Dictionnaire raisonné de la théorie du langage, Hachette, 1979
} 
Au reste, il faudra se pénétrer de cette vérité que la réflexion sur le langage n'est fructueuse que si elle porte d'abord sur les langues réelles. L'étude de ces organismes empiriques, historiques, que sont les langues demeure le seul accès possible à la compréhension des mécanismes généraux et du fonctionnement du langage.

Partant du réel des langues en usage, au jour le jour, la linguistique générale s'élève pour augmenter son champ de vision et comprendre, par exemple, que

[...] tous les hommes inventent leur propre langue sur l'instant et chacun de façon distinctive, et chaque fois d'une façon nouvelle. Dire bonjour tous les jours de sa vie à quelqu'un, c'est chaque fois une réinvention. [...] quand il s'agit de phrases, ce ne sont plus les éléments constitutifs qui comptent, c'est l'organisation d'ensemble complète, l'arrangement original, dont le modèle ne peut avoir été donné directement, donc que l'individu fabrique (BENVENISTE, 1974, p. 19).

Autrement dit, pour comprendre que par delà un système de langue stable et référent pour tout parlant de tel système, chaque parlant parle via ce système à sa manière, dans sa situation, bref, passe nécessairement par une énonciation qui sera chaque fois un " événement " parfaitement prévisible $e t$ imprévisible. La linguistique générale est une anthropologie.

Comme l'écrivent les éditeurs du livre d'hommage à Émile Benveniste Langue, discours, société, paru au Seuil en 1975, dans leur présentation,

Benveniste est un des trop rares linguistes qui ont toujours refusé de s'enfermer dans une conception étroite de leur discipline. Par ses propres travaux autant que par son influence directe ou indirecte, il en est venu ainsi à marquer et à renouveler profondément des disciplines voisines [...] anthropologie, mythologie, psychanalyse et théorie littéraire.

Dans son article "La fonction prédicative et le sujet parlant ", Julia Kristeva insiste sur ce point :

La linguistique, ce fameux " pilote " des sciences humaines, n'en continue pas moins de garder sa cohérence, appuyée sur la langue-contrat social fondamental ; mais elle s'ouvre à l'étude des mythes, des institutions et de l'inconscient, pour suggérer que leur isolement en "disciplines " séparées est injustifiable et sujet à refonte $[\ldots]$ La découverte de Benveniste est la découverte $\mathrm{du}$ procès signifiant en tant que matérialité hétérogène, multivalente : c'est une totalité, pour reprendre le terme hégélien qu'il emploie dans la préface de sa thèse en 1935, mais une totalité infinie, 
contradictoire et qui n'oublie jamais qu'elle se constitue d'une limite interne : la langue [...] [Benveniste] ne produit pas de système, mais dans chaque système signifiant, il scrute la limite (la langue) qui le constitue (KRISTEVA, 1975, p. 230).

\section{Qu'en est-il aujourd'hui des Problèmes de linguistique générale? Pierre Nora précise :}

L'ouvrage est tiré aujourd'hui à près de 35000 exemplaire dans la "Bibliothèque des sciences humaines" et 95000 dans la collection "Tel".

Les problèmes de linguistique générale II, parus en 1974 auront eux aussi un beau succès, quoique moindre : 11000 dans la "Bibliothèque des sciences humaines", 60480 dans la collection "Tel".

Voilà pour les chiffres qui disent, en matière d'édition, on le sait, beaucoup. Ceux-là disent que les Problèmes de linguistique générale sont lus, lus par les chercheurs et les linguistes. Michel Arrivé le confirme qui dit, dans un compte rendu des Dernières leçons d'Émile Benveniste:

Benveniste, aujourd'hui, est-il beaucoup lu ? Par les chercheurs, assurément : linguistes, de tout poil, mais aussi philosophes, ethnologues, "littéraires ", parfois analystes - à commencer par Lacan, qui pourtant l'aime peu - le citent très fréquemment dans leurs travaux. Les Problèmes de linguistique générale sont l'un des ouvrages de linguistique le plus souvent allégués, pas très loin du Cours de linguistique générale de Saussure, antérieur de quarante ans, et largement devant les Éléments de linguistique générale de Martinet, publiés en 1960, six ans avant les Problèmes. Pour l'instant, seul Chomsky - favorisé, certes, par la langue qu'il pratique - caracole loin devant les trois auteurs francophones, comme d'ailleurs devant Jakobson et Hjelmslev (ARRIVÉ, 2012, p. 121-125).

Mais il conclut : " cette lecture assidue des chercheurs s'accompagnet-elle pour Benveniste de la notoriété dans ce qu'on appelle « le grand public cultivé » ? Ce n'est pas certain. » (ARRIVÉ, 2012).

Il reste que, comme le remarque Roland Barthes,

[...] les livres de savoir, de recherche, ont aussi leur «style ». [Les Problèmes de linguistique générale] est d'une grande classe. Il y a une beauté, une expérience de l'intellect qui donne à l'œuvre de certains savants cette sorte de clarté inépuisable dont sont aussi faites les grandes œuvres littéraires. Tout est clair dans le livre de Benveniste, tout peut y être reconnu immédiatement pour vrai ; et cependant aussi, tout en lui ne fait que commencer (BARTHES, 1966, p. 816). 
Cette faiblesse de notoriété mondaine, pourrions-nous dire, n'empêche en rien que les Problèmes de linguistique générale demeure une référence et une source de réflexions. Le succès des Dernières leçons ${ }^{18}$ de Benveniste quelque peu inattendu - est, pour une part, la conséquence du succès toujours actuel des Problèmes de linguistique générale et, pour une autre part, la conséquence de leur relatif oubli.

\section{Références bibliografiques}

ARRIVÉ, Michel. Littérature, $n^{\circ}$ 168, décembre 2012, p. 121-125.

BARTHES, Roland. Situation du linguiste. In : Oeuvres complètes, 1966.

BENVENISTE, Émile. Structuralisme et linguistique. In : Problèmes de Linguistique Générale 2. Paris : Gallimard, 1974.

MILNER, Jean-Claude. Ibat Obscurus. In : Le périple structural, Paris : Seuil, 2002.

KISTEVA, Julia ; MILNER, Jean-Claude ; RUWET, Nicolas . Langue, discours, société : Pour Émile Benveniste. Paris : Seuil, 1975.

${ }^{18}$ Émile Benveniste, Dernières leçons. Collège de France 1968 et 1969, op. cit. 